BMJ Paediatrics Open

\title{
Serum concentrations of endothelial cell adhesion molecules and their shedding enzymes and early onset sepsis in newborns in Suriname
}

Rens Zonneveld, ${ }^{1,2}$ Rianne M Jongman, ${ }^{1,3,4}$ Amadu Juliana, ${ }^{2}$ Grietje Molema, ${ }^{1}$ Matijs van Meurs, ${ }^{1,3}$ Frans B Plötz ${ }^{5}$

To cite: Zonneveld $R$, Jongman RM, Juliana A, et al. Serum concentrations of endothelial cell adhesion molecules and their shedding enzymes and early onset sepsis in newborns in Suriname. BMJ Paediatrics Open 2018;2:e000312. doi:10.1136/ bmjpo-2018-000312

- Additional material is published online only. To view please visit the journal online (http://dx.doi.org/10.1136/ bmjpo-2018-000312).

Received 16 May 2018 Revised 30 July 2018 Accepted 1 August 2018
Check for updates

\section{(c) Author(s) (or their} employer(s)) 2018. Re-use permitted under CC BY-NC. No commercial re-use. See rights and permissions. Published by BMJ.

For numbered affiliations see end of article.

\section{Correspondence to} Dr Rens Zonneveld; rens. zonneveld@gmail.com

\begin{abstract}
Background Early onset sepsis (EOS) is defined as onset of sepsis within 72 hours after birth. Leucocyte-endothelial interactions play a pivotal part in EOS pathophysiology. Endothelial cell adhesion molecules (CAMs) orchestrate these interactions and their soluble isoforms (sCAMs) are released into the vasculature by enzymes called sheddases.
\end{abstract}

Purpose This study was undertaken to explore further the pathophysiology of EOS and to investigate the potential of SCAM and their sheddases as potential biomarkers for EOS.

Methods Stored serum aliquots were used from 71 Surinamese newborns suspected of EOS and 20 healthy newborns from an earlier study. Serum had been collected within 72 hours after birth and six $(8.6 \%)$ newborns had a positive blood culture with gram-negative pathogens. Concentrations of sCAMs sP-selectin, sE-selectin, soluble vascular cell adhesion molecule-1, intercellular adhesion molecule- 1 and platelet and endothelial cell adhesion molecule-1, sheddases matrix metalloproteinase-9 (MMP9) and neutrophil elastase (NE) and sheddase antagonist tissue-inhibitor of metalloproteinases-1 (TIMP-1) were measured simultaneously with Luminex and ELISA. Results MMP-9 and TIMP-1 levels were measured in serum of $n=91$ newborns and SCAMs and NE levels in serum of $n=80$ newborns, respectively. We found no differences in median concentrations of SCAMs, MMP-9 and TIMP-1 or NE between blood culture positive EOS, blood culture negative EOS and control groups at start of antibiotic treatment.

Conclusions Our data indicate that serum concentrations of SCAMs and their sheddases have no clinical utility as biomarkers for EOS.

Trial registration number NCT02486783. Results

\section{INTRODUCTION}

Early onset sepsis (EOS) in newborns within 72 hours after birth remains a clinical challenge with high morbidity and mortality. ${ }^{1-3}$ The majority of global neonatal deaths due to EOS occur in developing countries. ${ }^{4}$ The diagnosis of EOS is complicated, resulting in late recognition or overtreatment of

\section{What is already known on this topic?}

Recently, we established an association of the Angiopoietin (Ang)-1/Ang-2 disbalance with blood culture positive early onset sepsis (EOS) in newborns.

- The relationship between this Ang-1/Ang-2 disbalance and serum levels of soluble isoforms of cell adhesion molecules (sCAMs) and their sheddases is unclear.

\section{What this study hopes to add?}

The Ang-1/Ang-2 disbalance in blood culture positive EOS is not paralleled by increased levels of sCAMs and their sheddases.

- Levels of SCAMs and their sheddases are high after birth and do not discriminate EOS from healthy newborns.

newborns with antibiotics. These dilemmas arise because the pathophysiology of EOS is poorly understood.

A hallmark of sepsis pathophysiology is endothelial cell activation followed by leucocyte recruitment into tissues. ${ }^{5}$ The current model describes the occurrence of a shift in balance in Tie2 receptor ligands Angiopoietin (Ang)-1 and Ang-2 affecting endothelial integrity and increased expression of endothelial cell adhesion molecules (CAMs), in particular P-selectin, E-selectin, vascular cell adhesion molecule (VCAM-1) and intercellular adhesion molecule (ICAM-1) to facilitate this recruitment. ${ }^{67}$ These endothelial CAMs orchestrate leucocyte rolling on, adhesion to and diapedesis across the endothelium. ${ }^{78}$ Also, platelet and endothelial cell adhesion molecule (PECAM-1), expressed at endothelial cell junctions has a function in facilitating paracellular transmigration of leucocytes across the endothelium. ${ }^{9}$ After intravenous administration of endotoxin in healthy adults 
as a sepsis model, peak concentrations of Ang-2 prelude the release of soluble isoforms of cell adhesion molecules (sCAMs) into the systemic circulation. ${ }^{10}$ Endothelial CAMs are released through ectodomain shedding by enzymes called sheddases, in particular matrix metalloproteinase-9 (MMP-9) and neutrophil elastase (NE), released from granules in neutrophils. ${ }^{711}$ Both MMP-9 and NE prepare the extracellular matrix for transmigration of leucocytes into inflammatory sites. ${ }^{12}$ MMP-9 activity is balanced by sheddase antagonist tissue-inhibitor of metalloproteinases-1 (TIMP-1). ${ }^{12-14}$

Recently, we showed in a cohort of near term and term Surinamese newborns that a systemic disbalance in Ang-2/Ang-1 concentrations was associated with blood culture positive EOS. ${ }^{15}$ This study was undertaken to examine if this disbalance is paralleled by increased serum concentrations of sCAMs and sheddases in this cohort of newborns with EOS to explore further the pathophysiology of EOS. We hypothesised that sCAM and sheddases concentrations measured at start of antibiotic treatment for suspected EOS are higher in newborns with blood culture positive EOS than in healthy controls.

\section{MATERIALS AND METHODS}

\section{Study design, subjects and clinical protocol}

For this study, we used a Surinamese cohort of 20 healthy newborns and 71 newborns with suspected EOS from an earlier reported study. ${ }^{15}$ All newborns were included after admission between 1 April 2015 and 31 May 2016 to the neonatal care facility of Academic Pediatric Center Suriname at the Academic Hospital Paramaribo in Suriname. Included were newborns with a gestational age equal to or above 34 weeks in whom antibiotics were started within the first 72 hours of life for suspected EOS. Suspicion of EOS was based on the attending physicians decision to start antibiotic treatment. Informed consent was obtained from at least one parent for the use of residual serum and clinical information. The study protocol was made available on clinicaltrials.gov (NCT02486783) and was approved by the Surinamese Medical Ethical Board (VG-021-14A) including permission of one parent.

The management of these patients was described before. $^{15}$ In short, healthy control newborns and newborns suspected of EOS were included at start of antibiotic treatment within 72 hours after birth. At start of antibiotic treatment, blood was collected for separation and storage of serum. Controls were newborns without signs of infection receiving blood draws for hyperbilirubinaemia $(n=20)$. Newborns with suspected EOS receiving treatment with intravenous antibiotics were divided in two groups based on result from blood culturing: blood culture negative EOS $(\mathrm{n}=65)$ and blood culture positive $\operatorname{EOS}(n=6)$.

\section{Sample collection, preparation and analysis}

In the previous study, serum had been collected from whole blood collected after insertion of a venous cannula in newborns suspected of EOS and after capillary collection in controls. ${ }^{15}$ Frozen serum samples had been transported on dry ice to the Netherlands and aliquoted and frozen again on arrival. A stored aliquot was used for the measurement of sP-selectin, sE-selectin, vascular cell adhesion molecule-1 (sVCAM-1), sICAM-1 and sPECAM-1 using the Human Magnetic Bead Adhesion 6-plex panel performance assay (LHC0016M, Thermo Scientific, Waltham, Massachusetts, USA) according to the manufacturer's instructions. ELISA was used on the same aliquots for measurement of NE (HK319-02, Hycult Biotech, Uden, The Netherlands), MMP-9 (Quantikine DMP900, R\&D systems, Minneapolis, Minnesota, USA) and TIMP-1 (Quantikine DTM100, R\&D systems), each according to the manufacturers' instructions. For each molecule, a standard curve was established via which concentrations in neonatal serum were determined. Levels below or above the linear part of this standard curve were reported as the lowest or highest value of the standard curve, respectively. We measured intra-assay variation between plates used in the same assay by calculating coefficient of variation between levels of each molecule in samples from the same patient divided over those plates and accepted a maximum of $20 \%$.

\section{Statistical analysis}

A Kruskal-Wallis test with Dunn's correction for multiple comparisons was used for analysis between the blood parameters and the three groups (blood culture positive EOS, blood culture negative EOS and control groups). $<0.05$ were considered statistically significant. All analyses were done using Prism V.7.0a (Graphpad Software, San Diego, California, USA).

\section{RESULTS}

Demographic variables of the whole study cohort $(n=91)$ can be found in table 1 of Ref. 15. Of baseline characteristics, birth weight, age at presentation (between 0 and 72 hours after birth) and Apgar score at 5 min were distributed unevenly among the three groups $(p<0.05)$. Blood culture results revealed that 6 of 70 newborns with suspected EOS (8.6\%; $95 \%$ CI $1.9 \%$ to $15.3 \%)$ had a positive blood culture with gram-negative pathogens Klebsiella pneumoniae $(\mathrm{n}=2)$, Enterobacter cloacae $(\mathrm{n}=2)$ and Escherichia $\operatorname{coli}(\mathrm{n}=2)$. One newborn had EOS due to a spontaneous bacterial peritonitis. For $n=4$ other newborns, cause of EOS was unknown, but they presented with neonatal jaundice $(n=1)$, perinatal asphyxia $(n=1)$, meconium aspiration $(n=1)$ and hypoglycaemia $(n=1)$.

\section{Serum concentrations of soluble endothelial cell adhesion molecules and their sheddases}

Due to the limited amount of serum available, not all molecules could be measured in all samples. We were able to measure MMP-9 and TIMP-1 levels in serum of $\mathrm{n}=90$ newborns and sCAMs and NE levels in serum of 
$\mathrm{n}=80$ newborns, respectively. We found no differences in median concentrations of sCAMs (figure 1), MMP-9 and TIMP-1 (figure 2) or NE (figure 3) between blood culture positive EOS, blood culture negative EOS and control groups at start of antibiotic treatment within 72 hours after birth.

\section{DISCUSSION}

In this study, we investigated whether sCAMs and their sheddases circulate at higher concentrations in near and at term newborns with blood culture positive EOS at start of antibiotic treatment. In contrast to our hypothesis, none of the molecules showed any difference in serum concentrations between blood culture positive EOS, blood culture negative EOS and controls within 72 hours after birth. Our data indicate that serum concentrations of sCAMs and their sheddases have neither clinical utility as biomarkers for EOS nor to guide the start of antibiotic treatment.

Previously, we found evidence for endothelial cell activation in blood culture positive EOS in the same newborns used for this study, represented by a disbalance in Ang-2/Ang-1 ratio. ${ }^{15}$ Since the current data demonstrate that this disbalance was not paralleled by increased release of sCAM or sheddases in EOS, we conclude that endothelial CAM shedding is not or to a lesser extent involved in the pathophysiology of EOS. For interpretation of our data, we reviewed and summarised available data on sCAMs and sheddases in newborns with sepsis in online supplementary table $1 .{ }^{16-34}$ Comparison of our results with other existing data is complicated because of heterogenic makeup of chosen cohorts. Only one study reported a comparable cohort of near and at term newborns with suspected EOS within 72 hours after birth, in whom increased concentrations of SICAM- 1 and NE were associated with blood culture positive EOS. ${ }^{19}$ Other earlier studies compared concentrations of sCAMs in heterogenic cohorts consisting of newborns with different gestational and postnatal ages, either having EOS (based on varying definitions) or sepsis after 72 hours after birth (ie, late onset sepsis). This variation in inclusion criteria is an important confounding factor in the interpretation of the observed concentrations in septic and healthy newborns. Overall, our results are in line with these studies that show that clinical utility of sCAMs and sheddases in EOS is very limited.

In an earlier review by our group, we pooled published data on sCAM concentrations in newborns. ${ }^{7}$ Soluble CAM concentrations in the current study corresponded well with concentrations discussed in our review and those established in earlier studies in uninfected healthy newborns with similar gestational and postnatal age. ${ }^{72324}$ 29-32 However, MMP-9, TIMP-1 and NE concentrations were different and up to 4-fold, 2-fold and 10-fold higher, respectively, than those reported in earlier studies, ${ }^{171821} 222530$ which may have been due to other methods used (see limitations). Furthermore, our

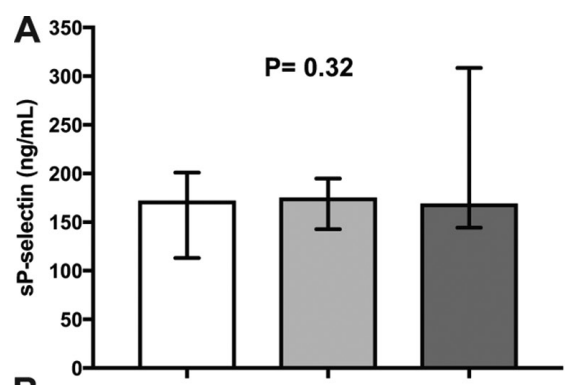

B
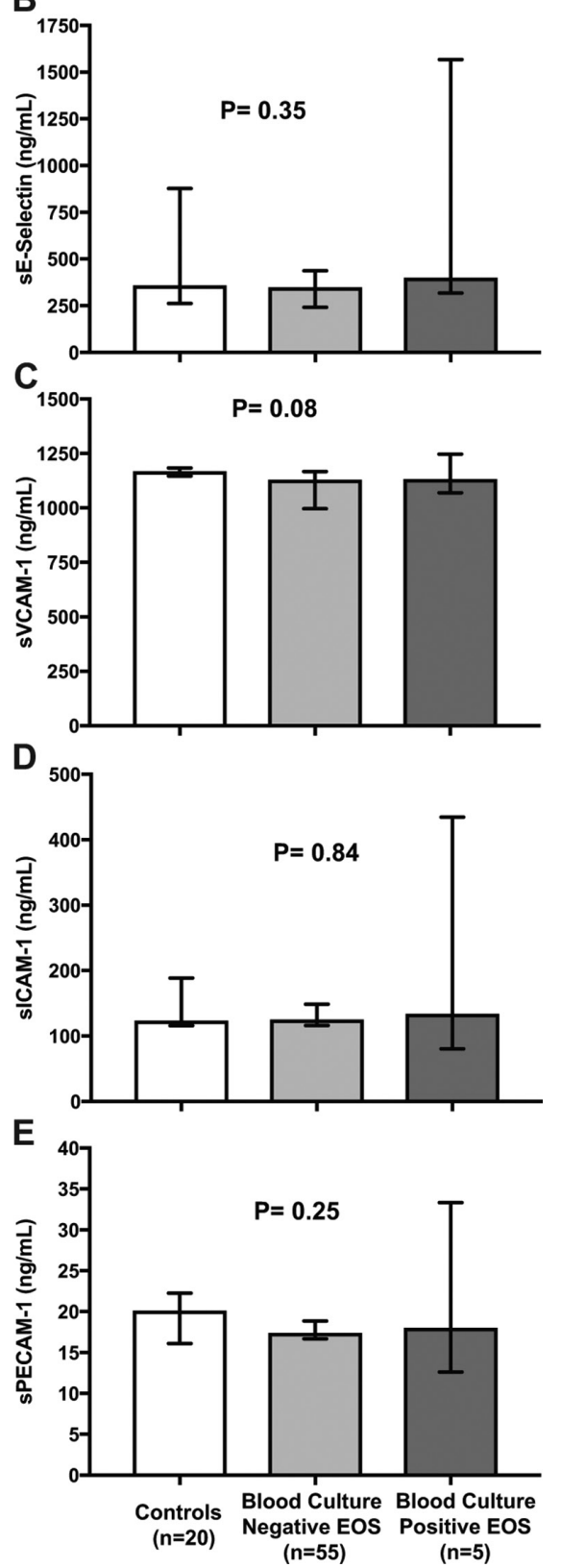

Figure 1 Circulating levels of endothelial adhesion molecules sP-selectin, sE-selectin, sVCAM-1, sICAM-1 and SPECAM-1 in Surinamese newborns. (A) sP-selectin; (B) sE-selectin; (C) sVCAM-1; (D) sICAM-1; (E) sPECAM-1. Bars represent median values and error bars $95 \% \mathrm{Cl}$. $\mathrm{P}<0.05$ were considered statistically significant. EOS, early onset sepsis; sICAM-1, soluble intercellular adhesion molecule-1; sPECAM-1; soluble platelet and endothelial cell adhesion molecule-1; sVCAM-1, soluble vascular cell adhesion molecule-1. 

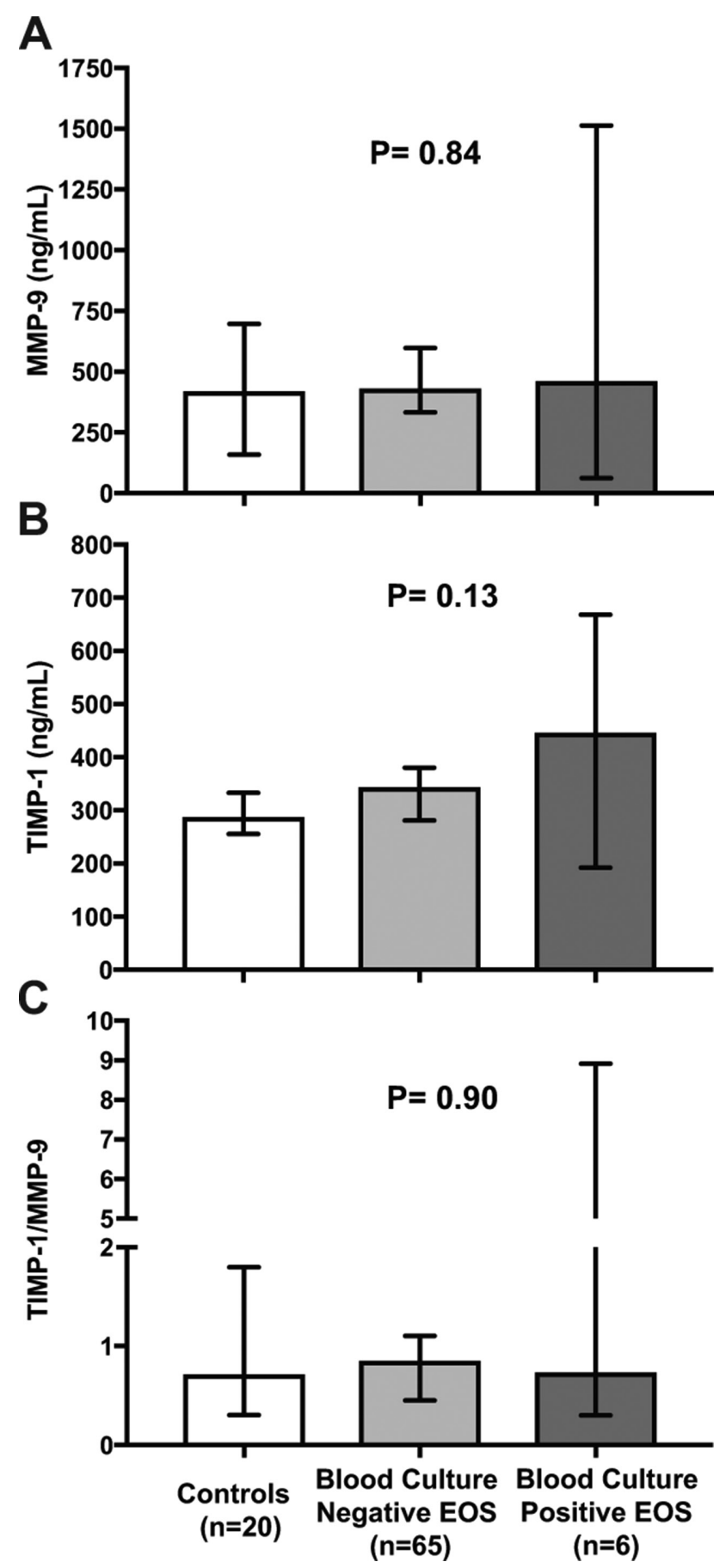

Figure 2 Circulating levels of MMP-9 and TIMP-1, and TIMP-1/MMP-9 ratios in Surinamese newborns. (A) MMP-9; (B) TIMP-1; (C) TIMP-1/MMP-9 ratios. Bars represent median values and error bars $95 \% \mathrm{Cl}$. $\mathrm{P}<0.05$ were considered statistically significant. EOS, early onset sepsis; MMP9, matrix metalloproteinase-9; TIMP-1, tissue inhibitor of metalloproteinase.

earlier review and earlier data indicated that significant age-related discrepancies exist in sCAM concentrations between newborns, children and adults. As an example, in at term newborns, sVCAM-1 concentrations in the first postnatal week were almost twice the concentrations in healthy adults and equally high compared with septic

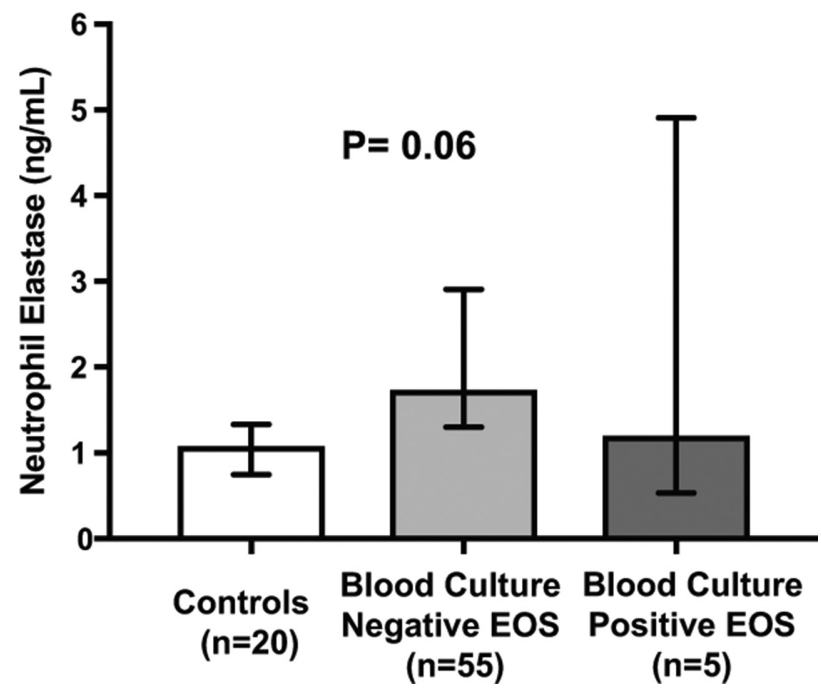

Figure 3 Circulating levels of neutrophil elastase in Surinamese newborns. Bars represent median values and error bars $95 \% \mathrm{Cl} . \mathrm{P}<0.05$ were considered statistically significant. EOS, early onset sepsis.

adults, suggesting that sVCAM-1 concentrations start of high in early newborn life and then decrease with increasing age. ${ }^{731}$ In our study, concentrations of sCAMs and sheddases during the first 3 days of life in our study remained stable, which was in contrast with earlier work in healthy newborns showing that sE-selectin decreased and sICAM-1 and SVCAM-1 increased between day 1 and 5 after birth, while sPECAM-1 levels did not change. ${ }^{29-32}$ Even though some discrepancies with earlier reports exist, overall one can conclude that these and our data indicate that concentrations of sCAMs and sheddases measured within 72 hours after birth are high and do not discriminate between septic and healthy newborns, which limits their use as biomarkers for early identification or exclusion of EOS.

Our and pre-existing data suggest that overall high sCAM and sheddase concentrations in newborns are the result of other perinatal factors than EOS. Several pathophysiological processes may explain this premise. Birth may induce a 'pro-adhesive' state of the endothelium leading to increased endothelial CAMs expression on, and shedding from, its surface. Additionally, the increase in overall leucocyte numbers and inflammatory activation of subsets associated with human birth, which was shown to be positively associated with increased perinatal stress ${ }^{35-37}$ may cause higher intensity of leucocyte-endothelial interactions and subsequent increases endothelial CAMs shedding. Aberrant adhesion of activated leucocytes to activated endothelium is associated with endothelial dysfunction and increased vascular permeability. ${ }^{38} 39$ Shedding of endothelial CAMs may then result in prevention of aberrant leucocyte adhesion on two complementary levels, namely (1) to lower endothelial CAMs density to prevent adhesion or promote de-adhesion of already adhering leucocytes and (2) to release circulating sCAMs that act as 'decoy receptors' to capture leucocytes in the 
vasculature to limit leucocyte-endothelial interactions. ${ }^{710}$ Whether this occurs in real life and what the contribution is to sCAM and sheddase concentrations in newborns remains unknown and could be studied in neonatal animal models. ${ }^{40-42}$

Our study has some limitations. First, sample size was relatively small. As a result, logistic regression analysis of other factors, such as maternal perinatal factors or method of birth, potentially influencing levels of sCAMs and sheddases, was precluded. Larger studies in countries such as Suriname, where the incidence of EOS is relatively high in comparison to Western countries, ${ }^{43}$ are necessary and can contribute to better insight in the vascular pathophysiology of EOS. Second, the use of serum in our study may have caused release of stored pools of MMP-9, TIMP-1 and NE from disrupted leucocytes during the clotting process, which could have accounted for higher levels of these molecules than reported in earlier studies. Last, repeated freeze-thaw cycles may have affected quality of serum samples with regards to reproducibility of NE concentrations.

In conclusion, our data indicate that serum concentrations of sCAMs and sheddases are not higher in Surinamese newborns with EOS versus controls at start of antibiotic treatment. Although concentrations may still increase significantly more in newborns with EOS, other mechanisms, such as perinatal stress during birth, may drive overall high concentrations in all newborns which precludes discrimination between septic and healthy newborns.

\section{Author affiliations \\ ${ }^{1}$ Department of Pathology and Medical Biology, University Medical Center Groningen, University of Groningen, Groningen, The Netherlands \\ ${ }^{2}$ Academic Pediatric Center Suriname, Academic Hospital Paramaribo, Paramaribo, Suriname \\ ${ }^{3}$ Department of Critical Care, University Medical Center Groningen, University of Groningen, Groningen, The Netherlands \\ ${ }^{4}$ Department of Anesthesiology, University Medical Center Groningen, University of Groningen, Groningen, The Netherlands \\ ${ }^{5}$ Department of Pediatrics, Tergooi Hospitals, Blaricum, The Netherlands}

Acknowledgements The research in this study was supported by the Thrasher Research Fund (TRF13064) (R. Zonneveld) and Tergooi Hospitals, Blaricum, The Netherlands. The authors acknowledge the efforts of all employees of the Clinical Laboratory of the Academic Hospital Paramaribo and the Central Laboratory of Suriname, Paramaribo, Suriname, for assistance with sample storage, handling and transport. We would like to thank Dr Ellen Tromp for assistance with the statistical analysis for the final version of this paper.

Contributors RZ, MvM, GM and FBP conceived and designed the study. RZ and AJ collected clinical data and collected the samples. RZ, RMJ and MvM prepared the samples and performed the sample analysis. RZ and MvM analysed the final database. RZ, MvM, GM and FBP drafted the manuscript. All authors coauthored and approved the final manuscript.

Funding The Thrasher Research Fund (TRF13064) and Tergooi Hospitals, Blaricum, The Netherlands.

Competing interests None declared.

Patient consent Obtained.

Ethics approval Surinamese Medical Ethical Board.

Provenance and peer review Not commissioned; externally peer reviewed.
Open access This is an open access article distributed in accordance with the Creative Commons Attribution Non Commercial (CC BY-NC 4.0) license, which permits others to distribute, remix, adapt, build upon this work non-commercially, and license their derivative works on different terms, provided the original work is properly cited, appropriate credit is given, any changes made indicated, and the use is non-commercial. See: http://creativecommons.org/licenses/by-nc/4.0/.

\section{REFERENCES}

1. van Herk W, Stocker M, van Rossum AM. Recognising early onset neonatal sepsis: an essential step in appropriate antimicrobial use. $J$ Infect 2016;72:S77-82.

2. Simonsen KA, Anderson-Berry AL, Delair SF, et al. Early-onset neonatal sepsis. Clin Microbiol Rev 2014;27:21-47.

3. Stoll BJ. Early-onset neonatal sepsis: a continuing problem in need of novel prevention strategies. Pediatrics 2016;138:e20163038.

4. Lawn JE, Cousens S, Zupan J. Lancet Neonatal Survival Steering Team. 4 million neonatal deaths: when? Where? Why? Lancet 2005;365:891-900.

5. Aird WC. The role of the endothelium in severe sepsis and multiple organ dysfunction syndrome. Blood 2003;101:3765-77.

6. Fiedler U, Augustin HG. Angiopoietins: a link between angiogenesis and inflammation. Trends Immunol 2006;27:552-8.

7. Zonneveld R, Martinelli R, Shapiro NI, et al. Soluble adhesion molecules as markers for sepsis and the potential pathophysiological discrepancy in neonates, children and adults. Crit Care 2014;18:204

8. Ley K, Laudanna C, Cybulsky Ml, et al. Getting to the site of inflammation: the leukocyte adhesion cascade updated. Nat Rev Immunol 2007;7:678-89.

9. Privratsky JR, Newman PJ. PECAM-1: regulator of endothelial junctional integrity. Cell Tissue Res 2014;355:607-19.

10. Kümpers P, van Meurs M, David S, et al. Time course of angiopoietin-2 release during experimental human endotoxemia and sepsis. Crit Care 2009;13:R64.

11. Garton KJ, Gough PJ, Raines EW. Emerging roles for ectodomain shedding in the regulation of inflammatory responses. J Leukoc Biol 2006;79:1105-16.

12. Elkington PT, O'Kane CM, Friedland JS. The paradox of matrix metalloproteinases in infectious disease. Clin Exp Immunol 2005;142:12-20.

13. Brew K, Nagase $\mathrm{H}$. The tissue inhibitors of metalloproteinases (TIMPs): an ancient family with structural and functional diversity. Biochim Biophys Acta 2010;1803:55-71.

14. Lorente L, Martín MM, Solé-Violán J, et al. Association of sepsisrelated mortality with early increase of TIMP-1/MMP-9 ratio. PLOS One 2014;9:e94318.

15. Zonneveld R, Jongman R, Juliana A, et al. Low serum angiopoietin-1, high serum angiopoietin-2, and high ang-2/ang-1 protein ratio are associated with early onset sepsis in surinamese newborns. Shock 2017;48:638-43.

16. Fattah MA, Omer AF, Asaif S, et al. Utility of cytokine, adhesion molecule and acute phase proteins in early diagnosis of neonatal sepsis. J Nat Sci Biol Med 2017;8:32-9.

17. Weitkamp JH, Guthrie SO, Wong HR, et al. Histological chorioamnionitis shapes the neonatal transcriptomic immune response. Early Hum Dev 2016;98:1-6.

18. Wynn JL, Guthrie SO, Wong HR, et al. Postnatal age is a critical determinant of the neonatal host response to sepsis. Mol Med 2015;21:1-504.

19. Sugitharini V, Prema A, Berla Thangam E. Inflammatory mediators of systemic inflammation in neonatal sepsis. Inflamm Res 2013;62:1025-34.

20. Edgar JDM, Gabriel V, Gallimore JR, et al. A prospective study of the sensitivity, specificity and diagnostic performance of soluble intercellular adhesion molecule 1 , highly sensitive $\mathrm{C}$-reactive protein, soluble E-selectin and serum amyloid $A$ in the diagnosis of neonatal infection. BMC Pediatr 2010;10:10-22.

21. Fukunaga S, Ichiyama T, Maeba S, et al. MMP-9 and TIMP-1 in the cord blood of premature infants developing BPD. Pediatr Pulmonol 2009;44:267-72.

22. Sunagawa $\mathrm{S}$, Ichiyama $\mathrm{T}$, Honda $\mathrm{R}$, et al. Matrix metalloproteinase-9 and tissue inhibitor of metalloproteinase-1 in perinatal asphyxia. Brain Dev 2009;31:588-93.

23. Figueras-Aloy J, Gómez-López L, Rodríguez-Miguélez JM, et al. Serum soluble ICAM-1, VCAM-1, L-selectin, and P-selectin levels as markers of infection and their relation to clinical severity in neonatal sepsis. Am J Perinatol 2007;24:331-8.

24. Sitaru AG, Speer CP, Holzhauer S, et al. Chorioamnionitis is associated with increased CD40L expression on cord blood platelets. Thromb Haemost 2005;94:1219-23. 
25. Schulz CG, Sawicki G, Lemke RP, et al. MMP-2 and MMP-9 and their tissue inhibitors in the plasma of preterm and term neonates. Pediatr Res 2004;55:794-801.

26. Edgar D, Gabriel V, Craig A, et al. A low serum sICAM-1 level may assist in the exclusion of neonatal infection. Biol Neonate 2002;81:105-8.

27. Apostolou M, Dimitriou H, Kaleyias J, et al. Levels of soluble ICAM1 in premature and full-term neonates with infection. Mediators Inflamm 2002;11:95-8.

28. Døllner H, Vatten L, Austgulen R. Early diagnostic markers for neonatal sepsis: comparing C-reactive protein, interleukin-6, soluble tumour necrosis factor receptors and soluble adhesion molecules. $J$ Clin Epidemiol 2001;54:1251-7.

29. Malamitsi-Puchner A, Tziotis J, Mastorakos G, et al. Adhesion molecules in early neonatal life. Biol Neonate 2000;78:65-7.

30. Giannaki G, Rizos D, Xyni K, et al. Serum soluble E- and L-selectin in the very early neonatal period. Early Hum Dev 2000;60:149-55

31. Giannaki G, Xyni K, Rizos D, et al. Comparative study of serum soluble VCAM-1 and ICAM-1 levels in the early neonatal period. Acta Paediatr 1999;88:1413-4.

32. Phocas I, Sarandakou A, Giannaki G, et al. Soluble intercellular adhesion molecule-1 in newborn infants. Eur $J$ Pediatr 1998;157:153-6.

33. Berner R, Niemeyer CM, Leititis JU, et al. Plasma levels and gene expression of granulocyte colony-stimulating factor, tumor necrosis factor-alpha, interleukin (IL)-1beta, IL-6, IL-8, and soluble intercellular adhesion molecule-1 in neonatal early onset sepsis. Pediatr Res 1998;44:469-77.

34. Austgulen R, Arntzen KJ, Haereid PE, et al. Infections in neonates delivered at term are associated with increased serum levels of ICAM-1 and E-selectin. Acta Paediatr 1997:86:274-80.
35. Lim FT, van Winsen L, Willemze R, et al. Influence of delivery on numbers of leukocytes, leukocyte subpopulations, and hematopoietic progenitor cells in human umbilical cord blood. Blood Cells 1994:20:547-58. discussion 558-9.

36. Lim FT, Scherjon SA, van Beckhoven JM, et al. Association of stress during delivery with increased numbers of nucleated cells and hematopoietic progenitor cells in umbilical cord blood. Am J Obstet Gynecol 2000;183:1144-51.

37. Duijts L, Bakker-Jonges LE, Labout JA, et al. Perinatal stress influences lymphocyte subset counts in neonates. The generation $\mathrm{R}$ study. Pediatr Res 2008;63:292-8.

38. Beyrau M, Bodkin JV, Nourshargh S. Neutrophil heterogeneity in health and disease: a revitalized avenue in inflammation and immunity. Open Biol 2012;2:120134.

39. Brown KA, Brain SD, Pearson JD, et al. Neutrophils in development of multiple organ failure in sepsis. Lancet 2006;368:157-69.

40. Shapiro NI, Yano K, Sorasaki M, et al. Skin biopsies demonstrate site-specific endothelial activation in mouse models of sepsis. $J$ Vasc Res 2009;46:495-502.

41. Csonka K, Vadovics M, Marton A, et al. Investigation of $\mathrm{OCH} 1$ in the Virulence of Candida parapsilosis Using a new neonatal mouse model. Front Microbiol 2017;8:1197.

42. Molina-Berríos A, Campos-Estrada C, Lapier M, et al. Protection of vascular endothelium by aspirin in a murine model of chronic Chagas' disease. Parasitol Res 2013;112:2731-9.

43. Zonneveld R, Holband N, Bertolini A, et al. Improved referral and survival of newborns after scaling up of intensive care in Suriname. BMC Pediatr 2017;17:189. 